\title{
Proteomics Pipeline for Biomarker Discovery of Laser Capture Microdissected Breast Cancer Tissue
}

\author{
Ning Qing Liu • René B. H. Braakman • \\ Christoph Stingl • Theo M. Luider • \\ John W. M. Martens • John A. Foekens • Arzu Umar
}

Received: 14 March 2012 / Accepted: 1 May 2012 / Published online: 30 May 2012

(C) The Author(s) 2012. This article is published with open access at Springerlink.com

\begin{abstract}
Mass spectrometry (MS)-based label-free proteomics offers an unbiased approach to screen biomarkers related to disease progression and therapy-resistance of breast cancer on the global scale. However, multi-step sample preparation can introduce large variation in generated data, while inappropriate statistical methods will lead to false positive hits. All these issues have hampered the identification of reliable protein markers. A workflow, which integrates reproducible and robust sample preparation and data handling methods, is highly desirable in clinical proteomics investigations. Here we
\end{abstract}

Electronic supplementary material The online version of this article (doi:10.1007/s10911-012-9252-6) contains supplementary material, which is available to authorized users.

N. Q. Liu • R. B. H. Braakman · J. W. M. Martens •

J. A. Foekens $\cdot$ A. Umar $(\bowtie)$

Department of Medical Oncology and Daniel Den Hoed Cancer

Center, Erasmus University Medical Center,

Dr. Molewaterplein 50, Be-401, P.O. Box 2040, 3000 CA

Rotterdam, the Netherlands

e-mail: a.umar@erasmusmc.nl

N. Q. Liu

e-mail: n.liu@erasmusmc.nl

N. Q. Liu $\cdot$ J. A. Foekens $\cdot$ A. Umar

Netherlands Proteomics Center,

Rotterdam, the Netherlands

R. B. H. Braakman · J. W. M. Martens · J. A. Foekens · A. Umar Center for Translational Molecular Medicine,

Rotterdam, the Netherlands

C. Stingl • T. M. Luider

Department of Neurology, Erasmus University Medical Center, Rotterdam, the Netherlands

J. W. M. Martens · J. A. Foekens · A. Umar

Cancer Genomics Centre,

Rotterdam, the Netherlands describe a label-free tissue proteomics pipeline, which encompasses laser capture microdissection (LCM) followed by nanoscale liquid chromatography and high resolution MS. This pipeline routinely identifies on average $\sim 10,000$ peptides corresponding to $\sim 1,800$ proteins from sub-microgram amounts of protein extracted from $\sim 4,000$ LCM breast cancer epithelial cells. Highly reproducible abundance data were generated from different technical and biological replicates. As a proof-of-principle, comparative proteome analysis was performed on estrogen receptor $\alpha$ positive or negative $(\mathrm{ER}+/-)$ samples, and commonly known differentially expressed proteins related to ER expression in breast cancer were identified. Therefore, we show that our tissue proteomics pipeline is robust and applicable for the identification of breast cancer specific protein markers.

Keywords Breast cancer $\cdot$ High resolution mass spectrometry $\cdot$ Label-free proteomics · Data analysis · Estrogen receptor associated proteins

$\begin{array}{ll}\text { Abbreviations } & \\ \text { AGC } & \text { Automatic gain control } \\ \text { CVs } & \text { Coefficient of variations } \\ \text { ER+/- } & \text { Estrogen receptor } \alpha \text { positive/negative } \\ \text { FDR } & \text { False discovery rate } \\ \text { LCM } & \begin{array}{l}\text { Laser capture microdissection/ } \\ \text { microdissected }\end{array} \\ \text { LCM-CTRLs } & \begin{array}{l}\text { Laser capture microdissected } \\ \text { control samples }\end{array} \\ \text { LFQ } & \begin{array}{l}\text { Label-free quantitation } \\ \text { Linear ion trap/Orbitrap mass } \\ \text { LTQ-Orbitrap-XL }\end{array} \\ \text { spectrometer } \\ \text { ME-ANOVA } & \begin{array}{l}\text { Mixed-effect analysis of variance } \\ \text { model } \\ \text { Mass spectrometry }\end{array}\end{array}$




\begin{tabular}{|c|c|}
\hline $\mathrm{m} / \mathrm{z}$ & Mass-to-charge ratio \\
\hline nLC-MS/MS & $\begin{array}{l}\text { Nanoscale liquid chromatography } \\
\text { coupled to tandem mass spectrometry }\end{array}$ \\
\hline & Polyethylene naphthalate \\
\hline & Progesterone receptor \\
\hline TL & Whole tissue lysate \\
\hline -CTRLs & Whole tissue lysate control samples \\
\hline
\end{tabular}

\section{Introduction}

With the rapid development of high resolution mass spectrometry (MS), global screening of protein markers becomes feasible and is starting to play an important role in biomarker discovery [1]. Protein markers are more related to disease phenotype and are more targetable for therapy in comparison with transcriptome-based biomarkers. Hence, identification of sensitive and specific protein makers is of importance for clinical practice. However, to develop a reproducible workflow for the robust identification of such biomarkers, several important technical aspects have to be taken into account.

A challenge in reliable protein marker identification is the heterogeneity of tumor tissues. Tumor cells are almost always surrounded by stromal compartments and infiltrating cells and the percentage of epithelial tumor cells can vary dramatically between individual tumor samples. Laser capture microdissection (LCM) is a widely applied technique to isolate tumor cells from their surrounding tissues [2, 3], which allows enrichment of cells of interest and removes bias introduced by comparison of tumor samples with different morphology. Nevertheless, LCM is a laborious and timeconsuming procedure, which means that only limited number of cells can be collected from individual samples, and is therefore difficult to apply on large cohort of tumor tissues when a large number of tumor cells per sample are needed for a successful measurement. Furthermore, a highly sensitive proteomics platform is required to analyze proteome of LCM materials in depth. Nanoscale liquid chromatography coupled to tandem mass spectrometry (nLC-MS/MS) enables identification of $>1,000$ proteins from sub-microgram breast cancer tissue in a $3 \mathrm{~h}$ gradient, and makes it possible to apply LCM for large scale biomarker discovery [4].

Secondly, the human proteome exhibits a very large dynamic range in protein expression, while MS based analysis can cover at best up to 4-5 orders of magnitude. This directly leads to reduced reproducibility for measurements of lower abundant proteins, because their corresponding peptides will not be consistently observed through all measured MS datasets, even though these peptides are biologically present through all the experimental samples, which leads to a large percentage of missing data in MS based proteomics. Moreover, sample handling steps are often complicated and need to be carefully controlled [5]. Furthermore, label-free quantification (LFQ) is often used for global screening of biomarkers but the quantitative capacity of LFQ remains a question. All these obstacles make it difficult to identify clinically valuable protein markers using an MS based proteomics approach. Great efforts have been made to improve protocols for sample preparation [6-8], to create sensitive and confident methods for multiple peak alignment, protein quantification and database searching $[9,10]$, and to perform more robust and reliable statistical analyses [11-14], in order to reliably identify biomarkers. Taken together, a well-designed pipeline for proteomics based biomarker discovery can greatly improve reproducibility of sample preparation, produce more quantitative data, and therefore increase the possibility of identifying reliable and clinically relevant biomarkers.

Here we describe a robust label-free tissue proteomics pipeline that is applicable for breast cancer biomarker discovery. This pipeline identified on average $\sim 10,000$ peptides corresponding to $\sim 1,800$ proteins from as little as $\sim 4,000$ LCM breast cancer epithelial cells (corresponding to sub-microgram protein lysates). Obtained data were highly reproducible and quantitative, and allowed identification of more than 100 differentially expressed proteins between estrogen receptor $\alpha$ positive $(\mathrm{ER}+)$ and negative $(\mathrm{ER}-)$ breast tumor samples.

\section{Methods}

Tumor Tissues and Samples

Ten fresh frozen tumor tissues were selected from our liquid N2 bank, of which 5 were ER positive (ER+) and 5 were ER negative (ER-). ER and progesterone receptor (PR) status of 10 samples were determined by enzyme-linked immunosorbent assay. From one ER tumor sample, five laser capture microdissected control samples (LCM-CTRLs, biological replicates) were prepared using a previously described protocol $[5,7]$. From the same biological source, whole tissue lysate (WTL) was prepared and measured by nLC-MS/MS for 12 times (whole tissue lysate control samples, WTL-CTRLs, technical replicates). The remaining five ER+ and four ERsamples were used as experimental samples to identify differentially expressed proteins. Detailed sampling plan and experimental design is explained in Supplementary Fig. 1. This study was approved by the Medical Ethics Committee of the Erasmus Medical Center Rotterdam, The Netherlands (MEC 
02.953) and was performed in accordance to the Code of Conduct of the Federation of Medical Scientific Societies in the Netherlands.

\section{Isolation of Breast Tumor Epithelial Cells}

Cryosectioning and LCM were performed according to previously described protocol [7]:

1. Sterilize and hydrate polyethylene naphthalate (PEN) coated glass slide (Carl Zeiss MicroImaging, GmbH, Munich, Germany) under ultraviolet light for $30 \mathrm{~min}$;

2. Cut 4 to 68 - $\mu \mathrm{m}$ tissue cryosections and attach those sections on a PEN slide;

3. Fix tissue sections in ice-cold $70 \%$ ethanol, briefly dry slides for $30 \mathrm{~s}$ at room temperature, and then dehydrate in ice-cold $100 \%$ ethanol;

4. Dry PEN slide in room temperature and place it in a plastic slide container wrapped with aluminum foil or Parafilm and store the slide container at $-80{ }^{\circ} \mathrm{C}$;

5. Prior to LCM, defrost the PEN slide at room temperate for $5 \mathrm{~min}$ inside a sealed slide container;

6. Rinse the PEN slide in tap water for $15 \mathrm{~s}$, stain tissue sections in hematoxylin for $30 \mathrm{~s}$, blue staining in tap water for $15 \mathrm{~s}$, and finally dehydrate tissue sections in 50, 70, 95 and $100 \%$ ethanol for $15 \mathrm{~s}$ each and $60 \mathrm{~s}$ for the final $100 \%$ ethanol wash. A volume of $200 \mu \mathrm{l}$ Halt protease and phosphatase inhibitor cocktail $(100 \times$ concentration, Thermo Fischer Scientific Inc., Rockford, IL, USA) is added into $20 \mathrm{ml}$ of tap water, 50 and $70 \%$ ethanol, respectively;

7. Isolate tumor cells using a P.A.L.M. MicroBeam system, and collect $\sim 500,000 \mu \mathrm{m}^{2}$ (equivalent to $\sim 4,000$ tumor cells [8]) in ZEISS opaque adhesive caps (Carl Zeiss MicroImaging, GmbH, Munich, Germany);

8. Suspend captured cells in $20 \mu \mathrm{l}$ of $0.1 \%$ RapiGest surfactant (Waters Corp., Milford, MA, USA) in $50 \mathrm{mM}$ ammonium bicarbonate (SIGMA, Saint Louis, MO, USA) buffer, store sample at $-80^{\circ} \mathrm{C}$.

Tip: (1) An optimal range of tumor area per dissection is between 5,000 and $25,000 \mu^{2}$ to ensure successful catapulting; (2) Always check the entire tumor sections under the microscope at $5 \times$ magnification to ensure all LCM tumor pieces are collected in the adhesive cap; (3) After transferring captured tumor cells into a LoBind Eppendorf tube (Eppendorf, Hamburg, Germany), check the cap under microscope to ensure all LCM tumor cells are transferred into the LoBind Eppendorf tube.
In-Solution Tryptic Digestion

In-solution tryptic digestion was performed according to the instructions of the manufacturer and as previously described [5]:

1. Lyse cell suspension in $0.1 \%$ RapiGest buffer using an Ultrasonics Disruptor Sonifier II (Model W-250/W-450, Branson Ultrasonics, Danbury, CT) at $70 \%$ amplitude for $1 \mathrm{~min}$;

2. Denature proteins at $95{ }^{\circ} \mathrm{C}$ for $5 \mathrm{~min}$;

3. Reduce denatured proteins at $60{ }^{\circ} \mathrm{C}$ for $30 \mathrm{~min}$ by adding dithiothreitol (SIGMA, Saint Louis, MO, USA) to a final concentration of $5 \mathrm{mM}$;

4. Alkylate reduced proteins in the dark for $30 \mathrm{~min}$ by adding iodoacetamide (Thermo Scientific, Rockford, IL, USA) to a final concentration of $15 \mathrm{mM}$;

5. Digest unfolded proteins at $37{ }^{\circ} \mathrm{C}$ for $4 \mathrm{~h}$ using MSgrade porcine modified trypsin gold (Promega, Madison, WI, USA) at a 1:4 (enzyme/protein) ratio ( $400 \mathrm{ng}$ of protein can be extracted from $\sim 4,000$ microdissected cells [1], therefore $100 \mathrm{ng}$ of trypsin was used for digestion);

6. Acidify tryptic digests with $0.5 \%$ trifluoroacetic acid (SIGMA, Saint Louis, MO, USA) and incubate mixture at $37{ }^{\circ} \mathrm{C}$ for $30 \mathrm{~min}$ to terminate tryptic digestion and degrade RapiGest;

7. Centrifuge digests at $14,000 \mathrm{rpm}$ for $15 \mathrm{~min}$ at $4{ }^{\circ} \mathrm{C}$ to precipitate undissolved cellular debris and the insoluble fraction of hydrolyzed RapiGest;

8. Transfer supernatant into high performance liquid chromatography vials for nLC-MS/MS measurements.

\section{nLC-MS/MS Analysis}

Proteomic profiling was performed on an Ultimate 3000 nLC system (Dionex, Amsterdam, The Netherlands) connected online with a hybrid linear ion trap/Orbitrap mass spectrometer (LTQ-Orbitrap-XL, ThermoElectron, Bremen, Germany) following a slightly modified procedure as described previously [8].

1. Method of sample loading: A volume of $20 \mu \mathrm{l}$ (equivalent to $\sim 4,000$ cells or $\sim 400 \mathrm{ng}$ ) from each sample was loaded on a trap column (PepMap C18, $300 \mu \mathrm{m}$ I.D. $\times 5 \mathrm{~mm}, 5 \mu \mathrm{m}$ particle size, $100 \AA$ pore size; Dionex, Amsterdam, The Netherlands) for concentration and desalting using $0.1 \%$ trifluoroacetic acid (in water) as loading solvent at a flow rate of $20 \mu \mathrm{l} / \mathrm{min}$;

2. nLC systems and elution gradient: After sample loading, the trap column was switched online to directly

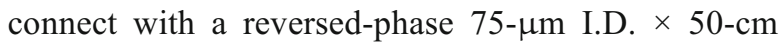


fused silica capillary column packed with $3-\mu \mathrm{m} \mathrm{C18}$ particles (PepMap, Dionex, Amsterdam, The Netherlands). Peptides were gradually eluted out at a flow rate of $250 \mathrm{nl} / \mathrm{min}$ at $40{ }^{\circ} \mathrm{C}$ column temperature using the following binary gradient: the gradient started with $100 \%$ mobile phase A $\left(97.9 \% \mathrm{H}_{2} \mathrm{O}, 2 \%\right.$ acetonitrile, $0.1 \%$ formic acid) to $25 \%$ mobile phase B (80\% acetonitrile, $19.02 \% \mathrm{H}_{2} \mathrm{O}, 0.08 \%$ formic acid) over the first $120 \mathrm{~min}$, and then a steeper gradient was used to further increase mobile phase B to $50 \%$ in the next $60 \mathrm{~min}$;

3. MS systems and settings: The eluted peptides were directly sprayed with a voltage of $1.6 \mathrm{kV}$ into the on-line coupled LTQ-Orbitrap-XL MS using nano electro-spray ionization source equipped with a metal-coated nano-scale emitter (New Objective, Woburn, MA). Mass spectra were acquired over a mass-to-charge ratio $(\mathrm{m} / \mathrm{z})$ range $400-1,800 \mathrm{Th}$ at a resolving power of 30,000 at $400 \mathrm{~m} / \mathrm{z}$. Target of automatic gain control (AGC) was set at $10^{6}$ ions and lock mass set to $445.120025 \mathrm{~m} / \mathrm{z}$ (protonated $\left.\left.\left(\mathrm{Si}\left(\mathrm{CH}_{3}\right)_{2} \mathrm{O}\right)\right)_{6}\right)$ [15]. On the basis of this full scan, the top 5 intensive ions were consecutively isolated (AGC target set to $10^{4}$ ions) and fragmented by collisionally activated dissociation applying $35 \%$ normalized collision energy in the linear ion trap. Parent ions within a mass window of $\pm 5 \mathrm{ppm}$ were then excluded for MS/MS fragmentation for the next 3 min or until the precursor intensity fell below a signal-to-noise ratio of 1.5 for more than 10 scans (early expiration). Orbitrap full scan spectra and ion trap MS/MS fragmentation spectra were acquired partially simultaneously (preview mode for FTMS master scan enabled).

\section{Database Searching}

The recorded MS spectra were analyzed by MaxQuant Software (version 1.1.1.36) [9].

1. Database searching and identification: The initial search was limited to a mass window of $7 \mathrm{ppm}$ and a fragment mass window of $0.5 \mathrm{Th}$. To construct an MS/MS peak list file, up to top 8 peaks per $100 \mathrm{Da}$ window were extracted and submitted to search against a concatenated forward and reverse version of the UniProtKB/Swiss-Prot human database (generated from version 2011_03, human taxonomy, 20,287 entries). Carbamidomethylation of cysteines was defined as fixed modification, while protein $\mathrm{N}$-terminal acetylation and methionine oxidation were defined as variable modifications for database searching. Also, an option of second identifications was selected to allow identification of co-eluting peptides with second highest searching score from a subset of MS/MS spectra. The cutoff of false discovery rate (FDR) for peptide and protein identification was set to 0.01 , and only peptides with $\geq 7$ amino acid residues were allowed for identification. In addition, at least one unique peptide was required to identify a protein;

2. Protein quantification and multiple peak alignment: LFQ was performed by MaxQuant software on identified razor and unique peptides in order to properly quantify identified proteins. Razor peptides are nonunique peptides assigned to the protein group identified by most other peptides [16], which follows "occam's razor" principle. Detailed methodology of LFQ algorithm was previously described in [17]. The "match between the runs" option was chosen to match the same accurate masses between multiple $\mathrm{nLC}-\mathrm{MS} / \mathrm{MS}$ runs within a retention time window of $2 \mathrm{~min}$.

\section{Data Processing and Statistical Analysis}

Data processing after MaxQuant data analysis was divided into two parts and performed as follows. A flow chart summarizes the complete strategy of statistical analyses used in our data handling pipeline (Supplementary Fig. 2).

1. $\log _{2}$ transformation, normalization and filtering of the data:

1.1. Peptide abundances given in the "peptides.txt" file generated by MaxQuant were first $\log _{2}$ transformed and then median peptide abundances in individual samples were centered;

1.2. Protein abundances normalized by LFQ algorithm integrated in MaxQuant were $\log _{2}$ transformed for further analyses. Label free algorithm takes the maximum number of identified peptides between any two samples and compares the intensity of these peptides to determine peptide ratios. Protein abundance is computed using median values of all peptide ratios of certain protein [17];

1.3. Peptides reserved for further analysis adhered to following criteria: (a) peptides were unique to one protein group, (b) sequences were not recognized as reversed sequences of all peptides in the database, and (c) peptides with large percentage of missing data were excluded from mixed-effect analysis of variance (ME-ANOVA) analysis. Due to the small sample cohort in this study, only peptides with abundance data in at least 5 
(50\%) observations out of 9 samples were included in the ME-ANOVA analysis. In case of larger sample cohorts (e.g. $n>60$ ), the threshold for peptide inclusion could be set to a minimum of $30 \%$ observations;

2. Statistical analysis:

In this part of data handling, we took two separate statistical approaches. Both of the two approaches were composed of pre-selection step (more sensitive, but less stringent) and refinement step (less sensitive, but more stringent). The preselection steps were used to find proteins that show a trend in differential expression between two experimental groups and therefore reduced numbers of multiple testing occurring in the refinement steps. The refinement steps aided to discover the strongest putative markers in the discovery study. The first approach consisted of ME-ANOVA pre-selection and $t$-test refinement (Step 2.1-2.3), which enabled finding proteins that were expressed in most of experimental samples but had significantly different abundance levels between two experimental groups. Therefore we defined this difference as "abundance" difference. The second approach combined Fisher's exact test for pre-selection and $t$-test refinement (Step 2.4). It aimed to identify proteins preferentially expressed in one of the experimental groups but which were not necessarily detected in majority of the experimental samples, which was defined as "presence-absence" difference. However, low abundant proteins are often not reproducibly detected through the entire MS dataset due to undersampling issue of shotgun proteomics, even though these proteins are indeed present in all the samples. Therefore, some stably expressed low abundant species can be mistaken for putative markers only when presence and absence are taken into account instead of actual abundance of these proteins. To avoid high FDR, only the proteins that were also differentially expressed at the level of imputed protein abundances were regarded as putative candidates.

2.1. ME-ANOVA test was performed on filtered peptides from Step 1.3, according to a previously described method [12, 13, 18]. This model takes into account four types of bias that may be introduced during the experimental procedure, known as experimental, group, peptide, and random error, and tries to calibrate these biases to achieve maximal separation between different experimental groups. In this model, higher abundant peptides assigned to certain protein weigh more than their lower abundant counterparts in estimating protein abundance. In our study, maximum 10 most abundant peptides per protein were used to test significance of their assigned proteins using a robust linear regression model in MEANOVA. However, it is difficult to estimate different biological and technical variations between the clinical samples since those samples were not collected under experimental conditions, especially no technical replicate was used for nLC-MS/MS profiling. Therefore, this model is only suitable for pre-selection of putative markers, and an additional step of consolidation is required to find truly differentially expressed proteins between different experimental groups;

2.2. Type I error (false positive hits) introduced during multiple hypothesis testing was corrected for using Benjamini-Hochberg $p$-value adjustment [19]. In this way, differentially expressed proteins were found using a corrected $p$-value cutoff of 0.05 ;

2.3. Next to the ME-ANOVA test, an additional $t$-test was performed on pre-selected putative markers identified by ME-ANOVA using their protein abundances to further refine the putative protein candidates $(p<0.05$, permutation-based FDR $=$ $0.05)$;

2.4. In ME-ANOVA test and subsequently $t$-test, proteins present in only one of the experimental groups led to invalid test and therefore could not be captured. Therefore, a Fisher's exact test was performed on MS/MS counts of all identified proteins except those recognized as reversed sequence $(p<0.05)$. In this way, proteins present in (mainly) one of the experimental groups could be discovered. Furthermore, data imputation was performed on the abundance of these differentially expressed proteins to replace missing values by normal distribution, and a $t$-test was performed on the imputed abundances to confirm differentially expressed patterns of these proteins $(p<0.05$, permutation-based $\mathrm{FDR}=0.05$ );

2.5. Hierarchical clustering was performed on the abundance of differentially expressed proteins. For hierarchical clustering analysis, protein expression data were first centered based on their median abundances, followed by clustering both samples and proteins using Euclidean distance and complete linkage.

Note: The filtering steps were performed in Microsoft Excel 2010. DanteR (version 1.0.1.1) 
and Perseus (version 1.2.0.17) were used to perform different types of statistical analysis including $\log _{2}$ transformation, correlation plot, statistical tests, imputation, $p$-value adjustment, and volcano plot, while hierarchical clustering was executed using Cluster 3.0 and visualized in TreeView (version 1.1.5r2-win).

\section{Results and discussion}

In this study, we describe a robust tissue proteomics pipeline for biomarker discovery, which enables identification of ER associated proteins in human breast cancer. The entire pipeline is divided into two different stages. The first stage consists of all procedures that generate raw nLC-MS/MS profiling data, while the latter part includes both upstream (multiple peak alignment, peptide and protein identification, and quantification) and downstream (statistical analysis) data handling steps. A flow chart summarizes the basic structure of this pipeline (Fig. 1).

To evaluate reproducibility of our tissue proteomics pipeline, we first inspected the number of identified peptides and protein groups of different samples. On average, $10,792 \pm 275(x \pm s), 10,539 \pm 742$ and 10,374 \pm 491 peptides were identified corresponding to $1,869 \pm 40$, $1,776 \pm 98$ and $1,869 \pm 60$ protein groups in 12 WTLCTRLs, 5 LCM-CTRLs and 9 experimental samples, respectively, and identifications of razor and unique peptides in three types of samples were also roughly equivalent (Table 1). Furthermore, as expected, LCMCTRLs had larger coefficient of variations (CVs) (7.0, 7.3 , and $7.4 \%$ ) than WTL-CTRLs $(2.6,2.6$, and $2.6 \%)$ on peptide, razor peptide and unique peptide identifications, respectively (Table 1). Thus, our tissue proteomics pipeline was able to consistently identify similar numbers of peptides and proteins in the same type of tissue materials. Peptide identifications and their abundances in all the samples are listed in Supplementary table 1 and 2 , and protein identifications and their abundances in all samples are listed in Supplementary table 3 and 4.

Reproducibility of abundance data was first investigated through Pearson correlation of all LCM-CTRLs and WTL-CTRLs using their peptide abundances. On

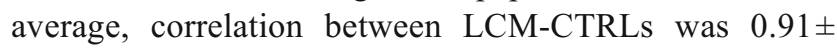
$0.02(x \pm s)$, and a slightly higher correlation was observed between WTL-CTRLs $(0.97 \pm 0.01)$ (Fig. 2a, left panel). This indicates a good reproducibility of our sample preparation protocol and nLC-MS/MS analyses. Correlation of peptide abundances between different experimental samples was lower $(0.72 \pm 0.04)$ (data not shown), which can be explained by both technical variation (e.g.: different tissue quality and different morphology) and, more importantly, biological variation (e.g.: inter- and intra-tumor heterogeneity). Next, we inspected reliability of estimated protein abundance. An average higher correlation was observed between all LCM-CTRLs $(0.94 \pm 0.01)$ and WTL-CTRLs $(0.98 \pm$ 0.01) using LFQ protein abundances (Fig. 2a, right panel) compared to correlation of peptide abundances. Moreover, we also observed a good Pearson correlation between protein abundance and MS/MS counts of the same sample in both LCM-CTRL $(0.84 \pm 0.03)$ and WTL-CTRL $(0.81 \pm 0.02)$ (data not shown). Thus, the LFQ algorithm properly computed and normalized protein abundance.
Figure 1 Flowchart summarizes the principle of label-free tissue proteomics pipeline. This technical platform is divided into two stages. The first stage generates nLCMS/MS raw data from tumor tissues, and the second part proposes a general data processing procedure used in MSbased label-free proteomics biomarker discovery study

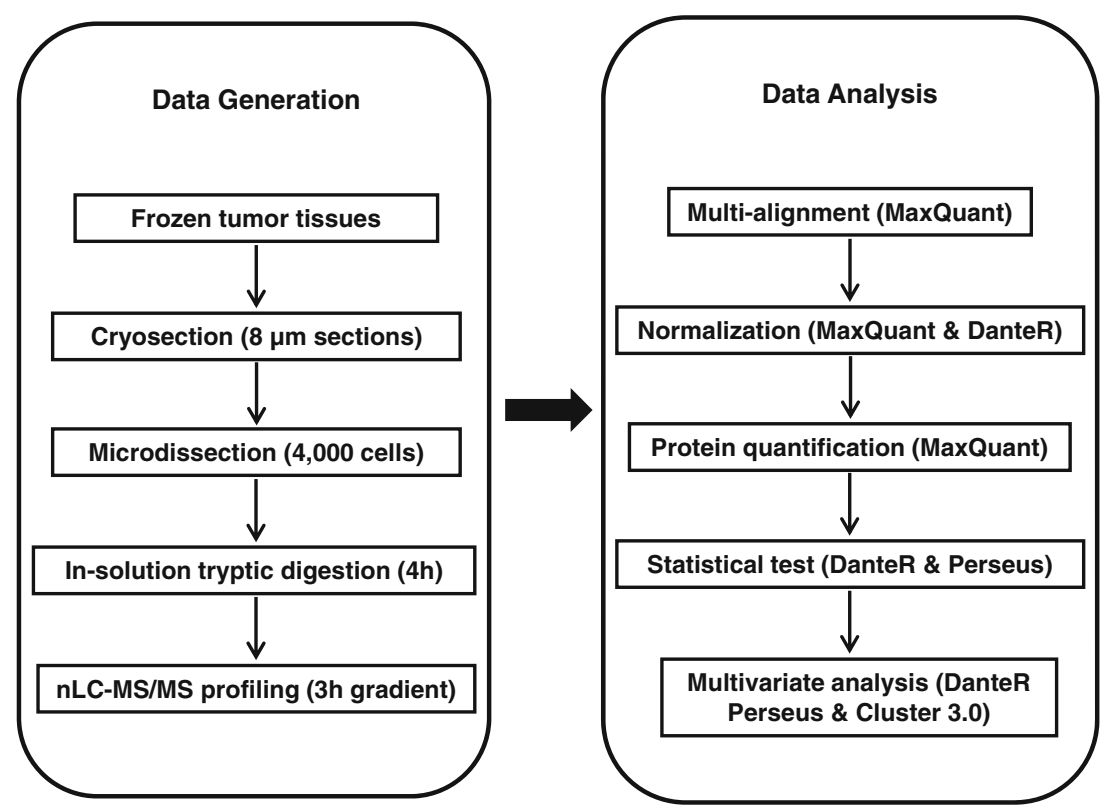


Table 1 Average numbers of identified peptides and protein groups

\begin{tabular}{lccr}
\hline Category & WTL-CTRL samples & LCM-CTRL samples & Experimental samples \\
\hline Total peptides & $10,792 \pm 275^{\mathrm{a}}(2.6 \%)^{\mathrm{b}}$ & $10,539 \pm 742(7.0 \%)$ & $10,374 \pm 491(4.7 \%)$ \\
Razor peptides & $488 \pm 10(2.0 \%)$ & $534 \pm 29(5.4 \%)$ & $483 \pm 17(3.5 \%)$ \\
Unique peptides & $9,664 \pm 254(2.6 \%)$ & $9,263 \pm 684(7.4 \%)$ & $9,217 \pm 472(5.1 \%)$ \\
Protein groups & $1,869 \pm 40(2.1 \%)$ & $1,776 \pm 98(5.5 \%)$ & $1,869 \pm 60(3.2 \%)$ \\
\hline
\end{tabular}

${ }^{\text {a }}$ Mean \pm Standard deviation $(x \pm s)$;

${ }^{\mathrm{b}}$ Percentages in brackets represent coefficient of variations of numbers of peptide and protein identification.

Quality of our data generation workflow was further inspected through reproducibility of peptide identifications in WTL-CTRLs, LCM-CTRLs or experimental samples. Peptide identifications in $\leq 33 \%, 34-66 \%$, and $\geq 67 \%$ and of all samples were considered to be of low, medium or high reproducibility, respectively. In WTL-CTRLs, LCM-CTRLs, and experimental samples $80 \%, 73 \%$, and $59 \%$ of peptides were identified with high reproducibility, whereas $9 \%, 14 \%$, and $23 \%$ of peptides were identified with medium reproducibility, and $11 \%, 13 \%$, and $18 \%$ with low reproducibility, respectively (Supplementary Fig. 3a, upper panel). At the protein level, $85 \%, 80 \%$, and $73 \%$ of identifications was observed in more that $66 \%$ of the samples (Supplementary Fig. 3a, lower panel). Furthermore, we observed that average CVs of the peptide abundances of WTL-CTRLs, LCM-CTRLs and experimental samples were $32.5 \% \pm 12.5 \%, 64.1 \% \pm 24.8 \%$ and $64.3 \% \pm$ $32.8 \%$, respectively (Supplementary Fig. 3b). Surprisingly, there was no significant difference in CVs of peptide abundances between LCM-CTRLs and experimental samples $(P>0.05)$, whereas there was a significant difference at the LFQ protein level $(33.3 \% \pm$ $25.3 \%$ and $81.3 \pm 51.5 \%$, respectively, $P<0.000$ ) (Supplementary Fig. 3b). In addition, CVs of WTL-CTRLs and LCM-CTRLs were overall lower in LFQ protein data $(21.1 \% \pm 19.9 \%$ and $33.3 \% \pm 25.3 \%$, respectively) than peptide data $(32.5 \% \pm 12.5 \%$ and $64.1 \% \pm 24.8 \%$, respectively), while CVs of experimental samples became larger after normalizing peptide abundance into protein abundance $(64.3 \% \pm 32.8 \%$ and $81.3 \pm 51.5 \%)$ (Supplementary Fig. 3b). These data indicate that the raw peptide abundance was properly normalized before further data mining, and further supports that LFQ algorithm properly normalized protein abundance. In conclusion, these observations suggest that reproducible data were generated using our tissue proteomics platform, and that upstream data analysis produced high quality data for further statistical analyses.

Next, WTL-CTRLs and LCM-CTRLs were compared to investigate the overlap in identified proteins. In total we identified 2,265 protein groups in 12 WTL-CTRLs and 5 LCM-CTRLs, of which $1,853(81.8 \%)$ were identified in both sample types (Fig. 2b). Only 241 $(10.6 \%)$ and $171(7.5 \%)$ protein groups were exclusively identified in either WTL-CTRLs or LCM-CTRLs, respectively (Fig. 2b). Unique proteins that were typically identified in WTL included most of major histocompatibility Class II molecules. These antigens are exclusively located on immune cells such as antigenpresenting cells and lymphocytes, which are only rarely

\section{a}

Peptide abundance

LFQ protein intensity

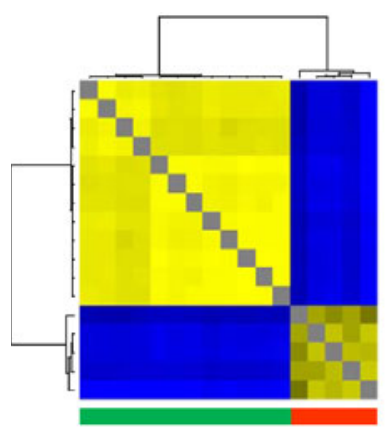

WTL-CTRL LCM-CTRL

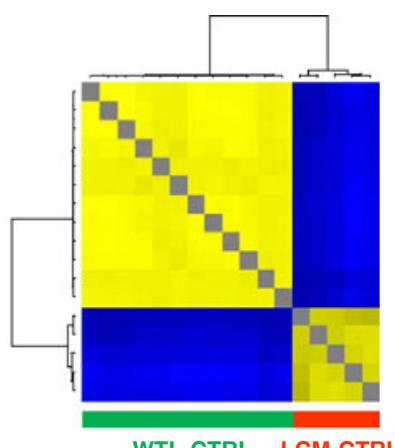

WTL-CTRL LCM-CTRL

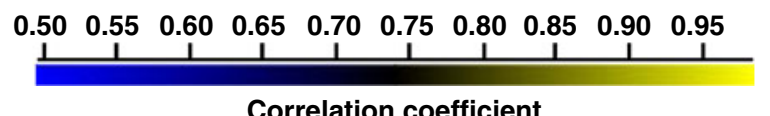

Correlation coefficient

b

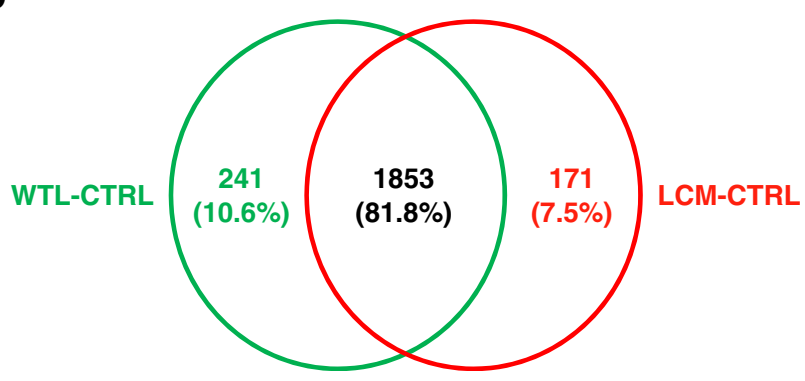

Figure 2 Application of label-free tissue proteomics pipeline to control and experimental breast cancer samples. a Pearson correlation of peptide and protein abundance between WTL-CTRLs and LCMCTRLs; b A Venn diagram reveals shared and unique identified protein groups in WTL-CTRLs (green circle) and LCM-CTRLs (red circle) 
Figure 3 Four breast cancer related proteins and their expression in ER+ and ERbreast cancer samples
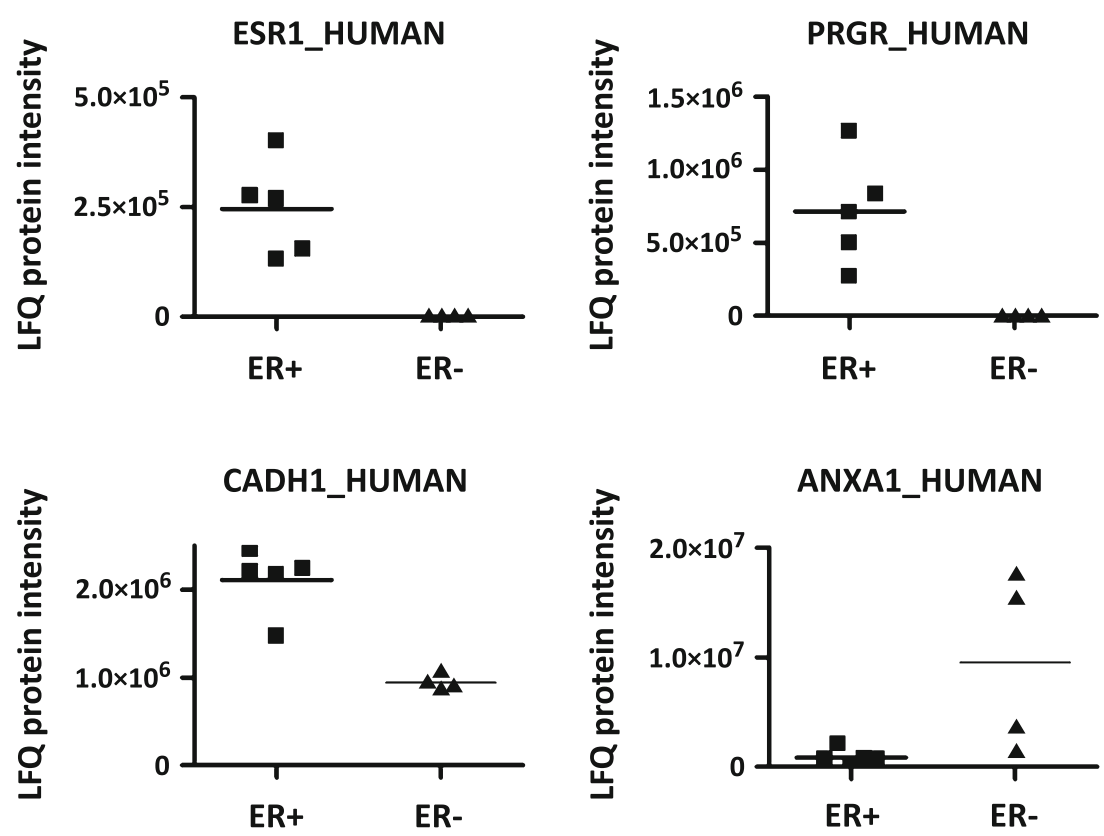

microdissected along with tumor cells and thus not often identified in LCM samples. Furthermore, extracellular matrix proteins such as some collagens present in the stromal compartment were identified in WTL samples. Some of these proteins are highly abundant and may have caused undersampling during the MS profiling, meaning that their lower abundant co-elutes in WTL samples escaped MS/MS fragmentation and remained unidentified, explaining $7.5 \%$ unique protein identification in LCM-CTRLs samples.

As a proof-of-principle, we applied the tissue proteomics and data handling pipeline to an experimental set of $5 \mathrm{ER}+$ and 4 ER- breast cancer tissues with the aim of identifying some known ER associated protein markers, in order to show that this platform can be used for large scale of biomarker discovery study. Protein abundance of ER (Entry name: ESR1_HUMAN), and downstream regulated proteins PR (Entry name: PRGR_HUMAN), Cadherin-1 (Entry name: CDH1_HUMAN) and Annexin A1 (Entry name: ANXA1_HUMAN), were investigated. As expected, ER and PR were completely absent in all 4 ER- samples (Fig. 3, upper panel), in concordance with in-house available enzyme-linked immunosorbent assay data from the same samples. Also, Cadherin-1 and Annexin A1 were elevated in ER+ and ER - samples (Fig. 3, lower panel), respectively. Cadherin-1, also known as E-cadherin, is well-known to mediate cell-cell adhesion, is important in breast cancer suppression, and is frequently down-regulated in ER- breast cancer cells [20]. Several clinical studies also showed that loss and aberrant expression of Cadherin-1 more frequently occurs in ER- breast cancer cases [21, 22], especially of the triple negative phenotype [23]. Also, Annexin A1 expression has been associated with breast cancer cell lines of the basal subtype [24], which are all ER-. In conclusion, these data indicate that the LFQ algorithm from MaxQuant was able to correctly determine relative protein abundance between different groups of breast cancer samples.

In order to reveal differentially expressed proteins between ER+ and ER- breast tumors, two different comparative proteome analyses were performed: (1) revealing quantitative differences (ME-ANOVA with $t$-test refinement); and (2) revealing proteins more frequently expressed in one of the experimental arms compared to the other (Fisher's exact test with $t$-test refinement). Using ME-ANOVA test, a total of 435 differentially expressed proteins were found between ER+ and ERbreast cancer samples $(p<0.05)$. ER + and ER- samples were well separated into two clusters using these 435 protein markers (Fig. 4a, left panel). However, the protein dendrogram (vertical axis) did not nicely fall into two clusters, indicating some low discriminatory proteins were also included in this clustering. To further refine the analysis to find the strongest markers, a $t$-test was used on $\log _{2}$ intensity values of 435 proteins to confirm differences between ER+ and ER- samples. With this more stringent refinement, 165 proteins were confirmed as differentially expressed proteins between ER+ and ERsamples $(p<0.05$, permutation-based FDR=0.05) (Supplementary table 5). Those 165 proteins formed a more solid protein dendrogram with two major arms (Fig. 4a, right panel). These findings suggest that ME-ANOVA with $t$-test refinement can reliably identify differentially expressed proteins between ER+ and ER- breast cancer samples. 
Figure 4 Differentially expressed proteins were discovered by different statistical analyses. a Hierarchical clustering separates ER+ and ERsamples using 435 (left panel) and 165 (right panel) differentially expressed proteins found by ME-ANOVA and refined using $t$-test; b Hierarchical clustering of 63

differentially expressed proteins between ER+ and ER- samples which were discovered using Fisher's exact test with $t$-test refinement, as well as expression of 4 differentially expressed proteins out of these 63 proteins

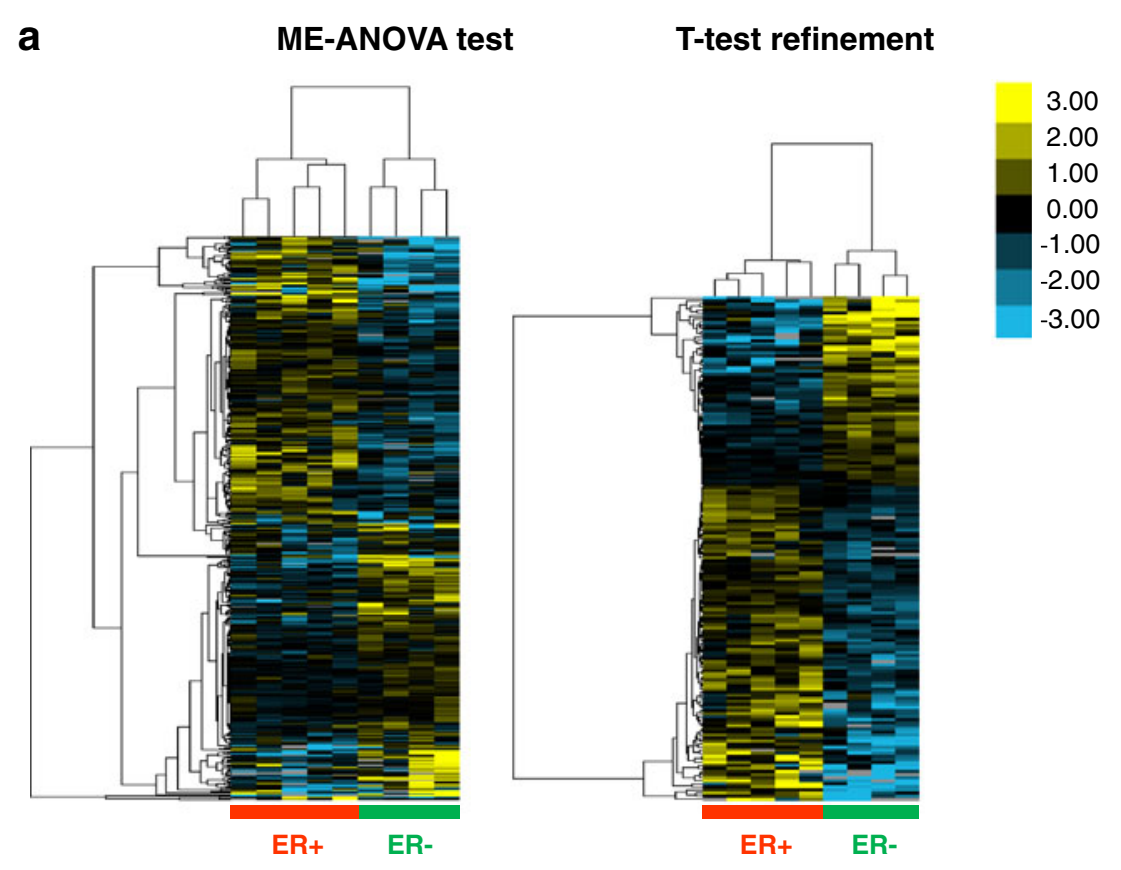

b Fisher's exact test with T-test refinement
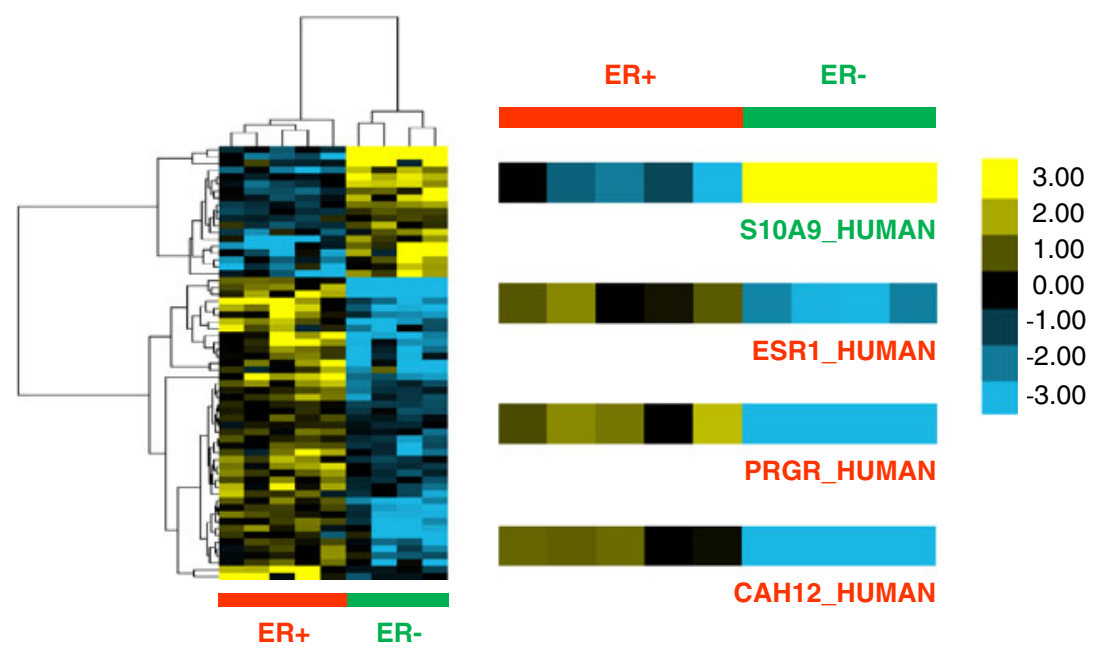

Additionally to reveal proteins more frequently present in one of the experimental arms, MS/MS counts of all identified proteins were subsequently analyzed by a Fisher's exact test in order to identify protein markers that are mainly present in one of the experimental groups. In total, 102 differentially expressed proteins were discovered using this approach. We further consolidated 63 proteins by performing a $t$-test on imputed $\log _{2}$ intensity of these 102 proteins using a $p$-value cutoff of 0.05 and permutationbased FDR of 0.05 (Supplementary table 6). ER+ and ER- samples could also be correctly classified using these 63 proteins (Fig. 4b). As expected, we found that ER and PR were enriched in ER+ breast cancer samples. Also, carbonic anhydrase 12 (Entry name: CAH12_HUMAN) and Protein
S100-A9 (Entry name: S10A9_HUMAN) were augmented in ER+ and ER- breast cancer samples, respectively (Fig. 4b). Carbonic anhydrase 12 was documented as an ER regulated protein in breast cancer, and expression level of this protein is highly positively correlated to expression level of ER [25, 26]. Protein S100-A9 has been associated with basal breast cancer which are typically ER- [27]. Moreover, 31 out of 63 differentially expressed proteins were also identified by ME-ANOVA test with $t$-test refinement with the same direction of regulation in ER+ and ER- samples (Supplementary table 7), which demonstrates validity of two types of statistical approaches, but both methods also provided complementary advantages in discovering putative markers. Therefore, ER, PR and some other ER associated proteins 
were only identified using combination of Fisher's exact test and imputation-based $t$-test refinement, indicating this approach is of added value to the quantitative statistical analysis.

In summary, we described a highly reproducible and robust label-free tissue proteomics pipeline for MSbased biomarker discovery. This platform produced high-quality MS data from as little as $\sim 4,000$ LCM breast tumor epithelial cells and reliably quantified protein abundance from observed peptide abundance. More importantly, it allowed identification of a large number of differentially expressed proteins between different experimental groups under investigation with relatively low FDR. Some of these differentially expressed proteins were previously described in literature as markers for ER+ or ER- breast cancer. Therefore, we conclude that this label-free tissue proteomics pipeline is suitable for clinical biomarker discovery.

Acknowledgments This work was financially supported by Netherlands Genomics Initiative/Netherlands Organisation for Scientific Research (NWO), and the Center for Translational Molecular Medicine, Breast CaRe project 030-104.

Open Access This article is distributed under the terms of the Creative Commons Attribution License which permits any use, distribution, and reproduction in any medium, provided the original author(s) and the source are credited.

\section{References}

1. Umar A, Kang H, Timmermans AM, Look MP, Meijer-van Gelder $\mathrm{ME}$, den Bakker MA, et al. Identification of a putative protein profile associated with tamoxifen therapy resistance in breast cancer. Mol Cell Proteomics. 2009;8(6):1278-94.

2. Emmert-Buck MR, Bonner RF, Smith PD, Chuaqui RF, Zhuang Z, Goldstein SR, et al. Laser capture microdissection. Science. 1996;274(5289):998-1001.

3. Espina V, Wulfkuhle JD, Calvert VS, VanMeter A, Zhou W, Coukos G, et al. Laser-capture microdissection. Nat Protoc. 2006;1(2):586-603.

4. Umar A, Luider TM, Foekens JA, Pasa-Tolic L. NanoLC-FT-ICR MS improves proteome coverage attainable for approximately 3000 laser-microdissected breast carcinoma cells. Proteomics. 2007;7(2):323-9.

5. Braakman RB, Tilanus-Linthorst MM, Liu NQ, Stingl C, Dekker LJ, Luider TM, et al. Optimized nLC-MS workflow for laser capture microdissected breast cancer tissue. J Proteom. 2012. doi:10.1016/j.jprot.2012.01.022.

6. Umar A, Dalebout JC, Timmermans AM, Foekens JA, Luider TM. Method optimisation for peptide profiling of microdissected breast carcinoma tissue by matrix-assisted laser desorption/ionisationtime of flight and matrix-assisted laser desorption/ionisation-time of flight/time of flight-mass spectrometry. Proteomics. 2005;5 (10):2680-8.

7. Braakman RB, Luider TM, Martens JW, Foekens JA, Umar A. Laser capture microdissection applications in breast cancer proteomics. Methods Mol Biol. 2011;755:143-54.

8. Stingl C, van Vilsteren FG, Guzel C, Ten Kate FJ, Visser M, Krishnadath KK, et al. Reproducibility of protein identification of selected cell types in Barrett's esophagus analyzed by combining laser-capture microdissection and mass spectrometry. J Proteome Res. 2011;10(1):288-98.

9. Cox J, Mann M. MaxQuant enables high peptide identification rates, individualized p.p.b.-range mass accuracies and proteome-wide protein quantification. Nat Biotechnol. 2008;26(12):1367-72.

10. Cox J, Neuhauser N, Michalski A, Scheltema RA, Olsen JV, Mann M. Andromeda - a peptide search engine integrated into the MaxQuant environment. J Proteome Res. 2011;10(4):1794-805.

11. Polpitiya AD, Qian WJ, Jaitly N, Petyuk VA, Adkins JN, Camp 2nd DG, et al. DAnTE: a statistical tool for quantitative analysis of -omics data. Bioinformatics (Oxford, England). 2008;24(13):1556-8.

12. Daly DS, Anderson KK, Panisko EA, Purvine SO, Fang R, Monroe ME, et al. Mixed-effects statistical model for comparative LC-MS proteomics studies. J Proteome Res. 2008;7(3):1209-17.

13. Karpievitch YV, Taverner T, Adkins JN, Callister SJ, Anderson GA, Smith RD, et al. Normalization of peak intensities in bottomup MS-based proteomics using singular value decomposition. Bioinformatics (Oxford, England). 2009;25(19):2573-80.

14. Karpievitch Y, Stanley J, Taverner T, Huang J, Adkins JN, Ansong $\mathrm{C}$, et al. A statistical framework for protein quantitation in bottomup MS-based proteomics. Bioinformatics (Oxford, England). 2009;25(16):2028-34.

15. Olsen JV, de Godoy LM, Li G, Macek B, Mortensen P, Pesch R, et al. Parts per million mass accuracy on an Orbitrap mass spectrometer via lock mass injection into a C-trap. Mol Cell Proteomics. 2005;4(12):2010-21.

16. Cox J, Matic I, Hilger M, Nagaraj N, Selbach M, Olsen JV, et al. A practical guide to the MaxQuant computational platform for SILACbased quantitative proteomics. Nat Protoc. 2009;4(5):698-705.

17. Luber CA, Cox J, Lauterbach H, Fancke B, Selbach M, Tschopp J, et al. Quantitative proteomics reveals subset-specific viral recognition in dendritic cells. Immunity. 2010;32(2):279-89.

18. Clough T, Key M, Ott I, Ragg S, Schadow G, Vitek O. Protein quantification in label-free LC-MS experiments. J Proteome Res. 2009;8(11):5275-84.

19. Benjamini Y, Hochberg Y. Controlling the false discovery rate- a practical and powerful approach to multiple testing. J R Stat Soc Ser B-Methodol. 1995;57(1):289-300.

20. Oesterreich S, Deng W, Jiang S, Cui X, Ivanova M, Schiff R, et al. Estrogen-mediated down-regulation of E-cadherin in breast cancer cells. Cancer Res. 2003;63(17):5203-8.

21. Putti TC, El-Rehim DM, Rakha EA, Paish CE, Lee AH, Pinder SE, et al. Estrogen receptor-negative breast carcinomas: a review of morphology and immunophenotypical analysis. Mod Pathol. 2005;18(1):26-35.

22. da Silva BB, dos Santos AR, Pires CG, Correa-Lima MA, PereiraFilho JD, dos Santos LG, et al. E-cadherin expression in estrogen receptor-positive and negative breast carcinomas of postmenopausal women. Eur J Gynaecol Oncol. 2010;31(1):90-3.

23. Kashiwagi S, Yashiro M, Takashima T, Nomura S, Noda S, Kawajiri $\mathrm{H}$, et al. Significance of E-cadherin expression in triple-negative breast cancer. Br J Cancer. 2010;103(2):249-55.

24. de Graauw M, van Miltenburg MH, Schmidt MK, Pont C, Lalai R, Kartopawiro J, et al. Annexin A1 regulates TGF-beta signaling and promotes metastasis formation of basal-like breast cancer cells. Proc Natl Acad Sci U S A. 2010;107(14):6340-5.

25. Gruvberger S, Ringner M, Chen YD, Panavally S, Saal LH, Borg A, et al. Estrogen receptor status in breast cancer is associated with remarkably distinct gene expression patterns. Cancer Res. 2001;61 (16):5979-84.

26. Barnett DH, Sheng S, Charn TH, Waheed A, Sly WS, Lin CY, et al. Estrogen receptor regulation of carbonic anhydrase XII through a distal enhancer in breast cancer. Cancer Res. 2008;68(9):3505-15.

27. McKiernan E, McDermott EW, Evoy D, Crown J, Duffy MJ. The role of $\mathrm{S} 100$ genes in breast cancer progression. Tumour Biol. 2011;32(3):441-50. 